\title{
Orbital metastasis in 68 years old female patient with papillary thyroid cancer presented in the year 2016
}

\begin{abstract}
Introduction: Ocular metastases of thyroid origin with infrequent about 5 to $6 \%$, usually grow slowly and clinically are painless, their aggressive forms are with local invasion or distant metástasis.

Case report: A 68-year-old female patient from Amazonas, with symptomatology of left retroocular pain, she had a disease period 3 months, presenting left ocular protrusion, with insidious onset and progressive course, for this reason performed a tomographic study where a left retro-ocular tumor was detected that displaced the eyeball forward. Also more than a year ago she presented a nodule in the right supraclavicular region of asymptomatic, progressive growth. It was evaluated by ophthalmology and a biopsy is requested, showed a thyroid papillary carcinoma metastasis. Later Subsequently it was thyroidectomized and it was suggested treatment with radioiodine.

Conclusion: Orbital metastases are infrequent however we should consider it as a differential diagnosis when we refer to the clinic of ocular proptosis, in addition an adequate management is important radioactive iodine therapy, thyroid stimulating hormone, chemotherapy and external radiotherapy.
\end{abstract}

Keywords: ocular metastasis, differentiated thyroid cancer
Volume 5 Issue 4 - 2018

\author{
Luis Carlos Araujo Cacha, ${ }^{1,2}$ Lesly Patricia \\ Ortiz Quispe 3 ,4 \\ 'Doctor of Medicine and Specialty in Nuclear Medicine, Peru \\ ${ }^{2}$ Peruvian Society of Nuclear Medicine and Molecular Imaging, \\ Peru \\ ${ }^{3}$ Student of the Alas Peruanas University School of Human \\ Medicine, Peru \\ ${ }^{4}$ Scientific and Academic Society of Medicine Students of Alas \\ Peruanas University-Lima-Peru, Peru
}

\begin{abstract}
Correspondence: Luis Carlos Araujo Cachay, Doctor of Medicine and Specialty in Nuclear Medicine, Peruvian Society of Nuclear Medicine and Molecular Imaging, Alas Peruanas University/UNMSM, Jr. Ayacucho 425 Dpto 505 Magdalena del Mar Lima-Perú, Peru, Tel 051997050458, Email laraujo@inen.sld.pe
\end{abstract}

Received: June 30, 2018 | Published: July 26, 2018

\section{Introduction}

Ocular metastases of thyroid origin with infrequent about 5 to $6 \%$, usually grows slowly and clinically are painless, although rare, their aggressive forms are with local invasion or distant metastasis, however it is important to keep them in mind to perform the proper management it is important to obtain a biopsy in addition to diagnostic help tests such as resonance or tomography to be able to assess the presentation in order to plan an adequate treatment.

\section{Clinical case}

\section{Current disease}

A 68-year-old woman, a native of Amazonas, from Lima, with symptomatology of left retroocular pain, has a disease time of approximately 3 months, presenting left ocular protrusion, with insidious onset and progressive course, for this reason they perform tomographic study where a retroocular ocular tumor was detected that displaced the eyeball forward.

Also more than a year ago she presented a nodule in the right supraclavicular region of asymptomatic, progressive growth. It was evaluated by ophthalmology and a biopsy is requested.

Ophthalmological Exam: Visual acuity: Right Eye: 20/30sc Left Eye: $20 / 100$ sc.

Left eye: Ocular proptosis. Ocular Motility: Partial restriction of the left eye to the left side.

A magnetic resonance image (MRI) weighted in T2 was obtained at the National Institute of Neoplastic Diseases that showed left and anterior lateral displacement of the eyeball with alteration of the signal in the bones of the left temporal and orbit (Figure 1).

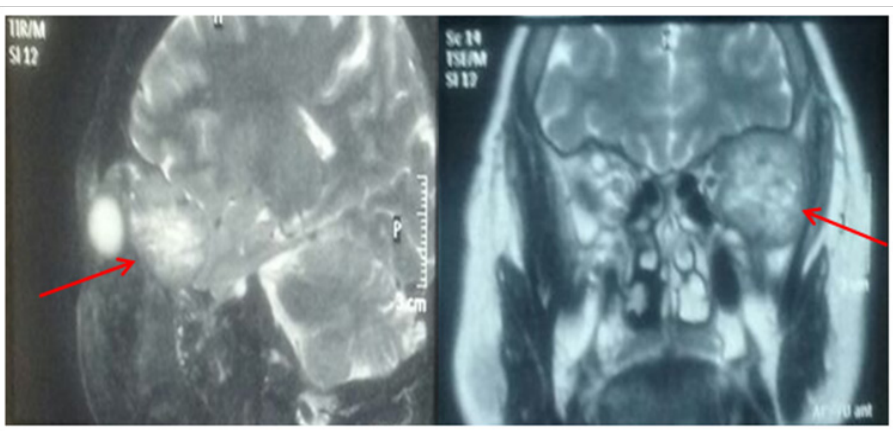

Figure I Magnetic resonance image revealed a extraconal tumor of the left orbit that causes mass effect on ipsilateral temporal region of bone origin (arrows).

A computed tomography (CT) scan showed extensive lytic bone metastatic involvement of the orbital and temporal bones with mass effect on the left orbital contents, causing loss of the normal sphericity of the eyeball and proptosis (Figure 2). Biopsy with fine needle aspiration under ultrasound guidance: Demonstrated metastasis of papillary carcinoma of thyroid.

With the results obtained, the patient underwent Surgery: total thyroidectomy+right neck dissection, whose pathological study showed: Papillary carcinoma of the Thyroid, follicular variant located in the right lobe of $1.3 \mathrm{~cm}$ well differentiated, with free surgical margins of goiter-associated neoplasia. Nine (9) positive nodes of 46 removed from group IV. Postoperative evolution was satisfactory suppressive treatment was started with levothyroxine $100 \mathrm{ucg} /$ day and after 2 months she died. 


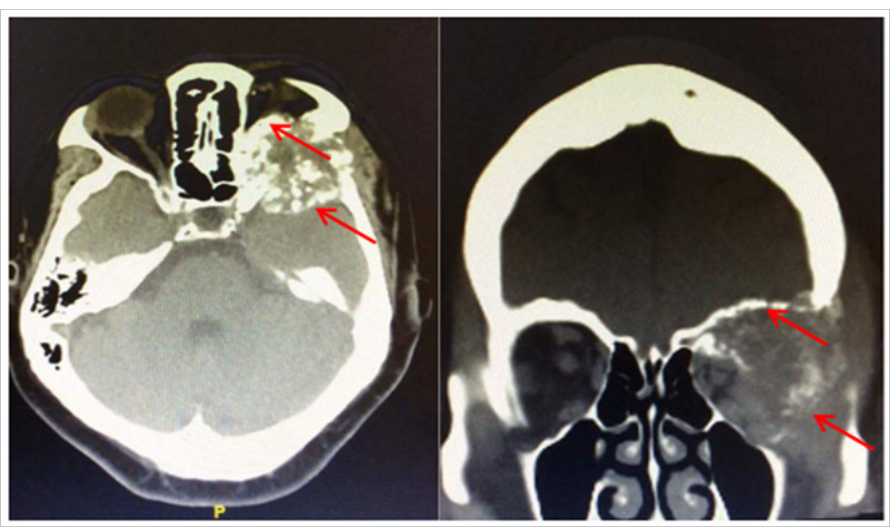

Figura 2 Extensive lytic bone metastatic lesion of the left orbit in contact with external wall (arrows) that infiltrates, destroys and invades the temporal fossa.

\section{Discussion}

Papillary thyroid carcinoma is the most common neoplasm of the thyroid represented by $85 \%$. It usually grows slowly and clinically they are painless, although rare, their aggressive forms are with local invasion or distant metastasis. Metastatic thyroid carcinoma rarely involves the orbit. ${ }^{1}$ The most frequent origin of orbital metastases are breast, prostate, kidney and melanoma. Follicular variant papillary thyroid carcinoma metastasizes via the blood, most commonly affecting bone and lung, and retro-orbital localization is uncommon around $5 \%$ to $6.5 \%$ of all orbital neoplasms. ${ }^{2}$

In relation to our case is the presentation of metastatic bone involvement in a patient with papillary histology with it is rarely observed since the few cases that are presented are with follicular histology. In most reported cases, skull metastases of thyroid cancer were located in the skull base and occipital area, ${ }^{3}$ but in our case, it was seen at the orbital bone. Most lesions of differentiated thyroid cancer with sensitive radioiodine, for this reason it is suggested that after thyroidectomy should receive therapy with radioiodine to decrease the likelihood of subsequent metastatic disease. ${ }^{4}$

Unilateral proptosis always needs an appropriate evaluation, especially in elderly patients, in search of a hidden neoplasia.
Unilateral proptosis as a manifestation of orbital metastasis from follicular thyroid cancer is rarely reported. Imaging tests with CT and MRI are of great help to perform a diagnostic orientation, although the definitive diagnosis is made by biopsy ${ }^{5}$ since there are no definitive radiological signs.

The treatment options are surgical resection of the primary and metastatic tumor, radioactive iodine therapy, thyroid stimulating hormone, chemotherapy and external radiotherapy. In our case, due to the patient death after 2 months of diagnosis, treatment with the radioiodine was not administered; nevertheless radioiodine therapy it could be useful in the management of primary disease, including proptosis resulting from the orbital metastasis. ${ }^{4}$

\section{Acknowledgements}

None.

\section{Conflict of interest}

The autor declares that their is no conflict of interest.

\section{References}

1. Ablis DP, Anthony HIA, Patrica DDM. Orbital metastasis as initial manifestation of a widespread papillary thyroid microcarcinoma. $B M J$ Case Reports. 2015;10(1136):1-4.

2. Dário RF, Lima GG, Ferreira FV, et al. Orbital Metastasis as Primary Clinical Manifestation of Thyroid Carcinoma - Case Report and Literature Review. Arq Bras Endocrinol Metabol. 2008;52(9):14971500 .

3. Akdemir I, Erol FS, Akpolat N, et al. Skull metastasis from thyroid follicular carcinoma with difficult diagnosis of the primary lesion. Neurologia Medico-Chirurgica. 2005;45(4):205-208.

4. PCN Okere, M Tushar. Retro-Orbital Metastasis from Differentiated Thyroid Carcinoma in a Radioiodine Therapy-Naïve Patient: Any Lesson Learned? Medical Principles and Practice. 2012;21(6):579-581.

5. Thattungal MA, Puthukuli $N$ Mini, Kalikavil $P$ Divya, et al. Thyroid follicular carcinoma presenting as intraorbital, intracranial, and subcutaneous metástasis. The American Journal of Surgery. 2010;199:72-74. 